\title{
Corrections aux volumes antérieurs
}

Correction au Sém. XXIV. Dans l'exposé de M. Emery "On two transfer principles...", une erreur tout à fait scandaleuse a été signalée par Serge Cohen, Page 416, dans la construction de l'extension $G$ de $F$, il faut utiliser une application $\pi$ de $Q$ dans $P$ qui préserve la première projection $\sigma: M \times N \longrightarrow M$ c'est-à-dire telle que $\sigma=\sigma \circ \pi$; or une telle $\pi$ n'existe que si la restriction de $\sigma$ à $P$ est surjective et submersive de $P$ sur $M$. Le plus simple est de rajouter cette hypothèse au théorème 4 ; elle est de toute façon déjà automatiquement vérifiée en tous points de la forme $\left(X_{t}(\omega), Y_{t}(\omega)\right)$ par l'existence même d'un morphisme de Schwartz contraint (hypothèse (i)) donc partout au-dessus de $X$. Cette erreur sera corrigée dans un travail à paraître de Cohen; le scandale annoncé plus haut vient de ce qu'une note en bas de page signale que cette partie de la démonstration prétend corriger une erreur antérieure dans une autre publication!

Complément au Sém. XXV. Dans l'exposé de M. Emery et G. Mokobodzki "Sur le barycentre d'une propriété dans une variété", la conjecture énoncée en dernière ligne de la page 232 (et dont le premier auteur porte seul la responsabilité) a été réfutée par un magnifique exemple de Wilfrid Kendall (à paraître, intitulé "The propeller"). 